\title{
Trajectory Planning for Emergency Landing of VTOL Fixed-Wing Unmanned Aerial Vehicles
}

\author{
Zhao Deng $\mathbb{D}$, Zhiming Guo $\mathbb{D}$, Liaoni $W u(\mathbb{D}$, and Yancheng You
}

UAV Lab, School of Aerospace Engineering, Xiamen University, Xiamen, China

Correspondence should be addressed to Liaoni Wu; wuliaoni@xmu.edu.cn

Received 31 August 2021; Revised 2 November 2021; Accepted 11 November 2021; Published 29 November 2021

Academic Editor: Fazlullah Khan

Copyright (c) 2021 Zhao Deng et al. This is an open access article distributed under the Creative Commons Attribution License, which permits unrestricted use, distribution, and reproduction in any medium, provided the original work is properly cited.

In recent years, inspired by technological progress and the outstanding performance of Unmanned Aerial Vehicles (UAVs) in several local wars, the UAV industry has witnessed explosive development, widely used in communication relay, logistics, surveying and mapping, patrol, surveillance, and other fields. Vertical Take-Off and Landing fixed-wing UAV has both the advantages of vertical take-off and landing of rotorcraft and the advantages of long endurance of fixed-wing UAV, which broadened its application field and is the most popular UAV at present. Recently, fixed-wing UAV failure analysis highlights that cruise engine shutdown is the most common reason for emergency landing, which is also a governing factor for Vertical Take-Off and Landing (VTOL) fixed-wing UAV failures. Nevertheless, the emergency landing trajectory of the latter UAV type after engine shutdown is different from that of the conventional fixed-wing UAVs due to the VTOL power system. Hence, spurred by the requirement of a safe emergency landing trajectory for VTOL fixed-wing UAVs, this paper develops an architecture capable of safe emergency landing for such platforms. The suggested method develops a particle dynamics model of the VTOL UAV and analyzes its aerodynamic characteristics utilizing Computational Fluid Dynamics (CFD) results. The UAV's trajectory is divided into three parts for enhanced planning. For the guidance stage, the initial position and heading angle are arbitrary. Hence, the Dubins shortest cross-range and the fastest descent trajectory are adopted to steer the UAV above the landing window quickly. The spiral stage comprises a conical and cylindrical part combined with a spiral descent trajectory of variable radius for energy management and landing course alignment. Given the limited energy storage of VTOL power systems, the landing stage exploits an optimal control trajectory problem solved by a Gaussian pseudospectral method, involving trajectory conventional landing planning, unpowered landing, distance optimal landing, and wind-resistant landing. All trajectories meet the dynamics constraints, terminal constraints, and sliding performance constraints and cover both 2-dimensional and 3-dimensional trajectories. A large number of simulation experiments demonstrate that the proposed trajectories manage broad applicability and strong feasibility for VTOL fixed-wing UAVs.

\section{Introduction}

Fixed-wing Unmanned Air Vehicles (UAVs) with Vertical Take-Off and Landing (VTOL) capabilities do not require a runway to take off and land, significantly reducing the risk of accidents during both these accident-prone flight stages. Additionally, VTOL platforms afford a broader application field carrying out missions in inaccessible environments [1]. Furthermore, VTOL UAVs involve a multirotor mode during take-off and landing, simplifying the operator's control. Accordingly, one-key autonomous take-off and landing are easier, improving reliability and safety during these flight phases, and afford nonprofessional and unskilled users to utilize UAVs [2, 3]. A VTOL UAV flight includes the multirotor, transition, and fixedwing stages, followed by a typical flight mission profile. A complete VTOL flight profile is illustrated in Figure 1, with the corresponding flight stages analyzed as follows: 


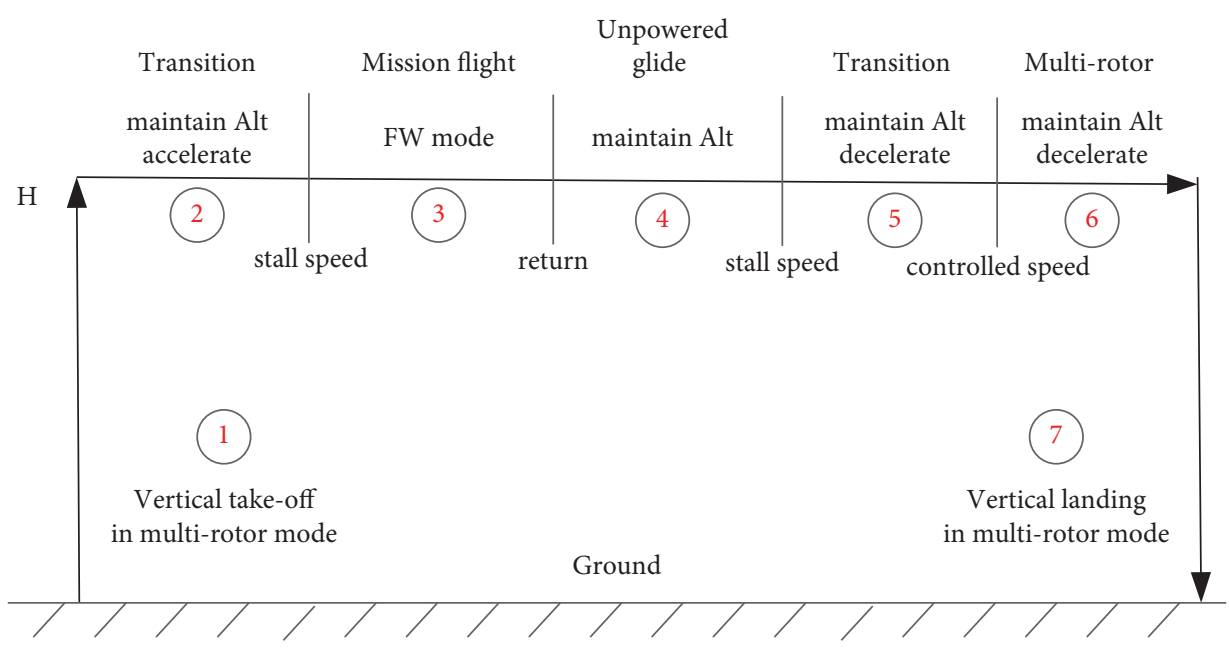

FIgURE 1: VTOL UAV flight profile.

Take-off: The UAV takes off vertically in the multirotor mode until it reaches a safe altitude. Then the horizontal cruise engine starts providing the UAV horizontal acceleration.

Accelerated transition: In the early stage, the velocity pressure is low, and thus the lift generated by the wing does not counterbalance gravity imposing the UAV to fly in the multirotor mode. When the airspeed is greater than the stall speed, the rotors stop working, and the UAV enters the cruise stage in the fixed-wing mode.

Deceleration transition: The UAV turns off the cruise engine, preserves its flying altitude, and decelerates. When the speed is less than the stall speed, the rotor system is activated, and the UAV continues to decelerate.

Landing: When the speed is below a threshold, for example, $0.5 \mathrm{~m} / \mathrm{s}$, the UAV lands vertically in the multirotor mode.

During the take-off, transition, and landing phases, the eight rotors generate lift force that balances gravity and controls attitude. If the UAV operating in multirotor mode needs to meet a certain level of wind resistance, the VTOL power must have a large margin (margin coefficient $P_{C}=\left(F_{M} / G\right) \geq 1.6$ ), and thus a Li-battery pack providing power to the rotors with additional power (capacity) is needed. On the contrary, rotors do not operate during the cruise phase, and the Li-battery pack is dead weight, significantly reducing the payload. Therefore, reducing the Li-battery pack weight is mandatory to meet the performance indicators, for example, wind resistance $[4,5]$.

Current solutions involve increasing the energy density of Lithium-ion batteries or develop a reasonable planning strategy to improve energy efficiency during the landing phase under the condition of limited energy reserve. The former solution is currently governed by a technical bottleneck, which is difficult to overcome in the foreseeable future. Therefore, it is necessary to study the landing trajectory to plan an emergency landing trajectory after engine shutdown or optimize the energy consumption of the limited energy stored in VTOL power systems.

In [6], Gaussian pseudospectral method (GPM) and arc transition methods are utilized to plan the trajectory of the initial descent phase for near-space glide vehicles, respectively. The simulation results highlight that all constraints can be satisfied using the two proposed methods. In addition to this, the arc transition method does not require the traditional nominal angle of attack design, which can be applied online with low computational cost. The work of [7] suggests a GPM to optimize the trajectory of a typical pointto-point mission for a high-altitude solar-powered fixedwing UAV, and the optimum path is compared with a current constant-altitude constant-velocity path. The results indicate that the proposed optimum path affords more energy profits, improving the battery pack's final charge state by $18.8 \%$. A $2 \mathrm{D}$ horizontal trajectory for an emergency landing of a large fixed-wing UAV without power is designed in [8]. This method utilizes the plane geometry position analysis and uses the principle of balanced glide to design the altitude trajectory section. Despite this method being theoretically sound, it lacks practical engineering verification. Reference [9] investigates the emergency return route trajectory for a VTOL fixed-wing UAV after engine shutdown without considering the trajectory from the landing window to the ground, which is crucial for VTOL UAVs or fixed-wing UAVs safe landing.

Most of the emergency landing trajectory planning studies consider traditional fixed-wing aircraft and rarely investigate the VTOL UAV. However, the emergency landing trajectory is critical for a VTOL UAV safely, and, most importantly, the trajectory is very different from the traditional fixed-wing aircraft. Hence, this paper fills this research gap by utilizing GPM to plan the landing trajectory by solving the minimum energy consumption and finite energy storage's longest or shortest flight distance problem. Meanwhile, the emergency landing trajectory after engine shutdown is also given. 
The rest of the paper is organized as follows: Section 2 mathematically models a fixed-wing VTOL UAV, while Section 3 platforms aerodynamic analysis based Computational Fluid Dynamics (CFD) simulation. Section 4 introduces the states and control constraints, and Section 5 challenges the proposed method on several flight trajectories. Finally, Section 6 concludes this work.

\section{Mathematical Model}

The experimental platform is a fixed-wing UAV with a double-tail brace structure, on which eight rotors are added which provide VTOL capabilities (Figure 2).

In our trials, we consider the dynamic equation of the center of mass in the flight path coordinate system and the UAV's kinematic equation in the Earth-surface reference frame. The UAV's center of mass motion can be described utilizing the momentum theorem:

$$
m \frac{\mathrm{d} V}{\mathrm{~d} t}=F,
$$

where $m$ is UAV's mass, $V$ represents the velocity vector, and $F$ is the external force vector at the center of mass. The centroid dynamics equation projected on the flight path coordinate system can be expressed as [10]

$$
\left[\begin{array}{c}
\dot{V} \\
V \dot{\chi} \cos \gamma \\
-\dot{\gamma} V
\end{array}\right]=\frac{L_{k a}}{m}\left(\begin{array}{c}
-D-D_{M} \\
C \\
-L
\end{array}\right)+\frac{L_{k b}}{m}\left(\begin{array}{c}
T \\
0 \\
-L_{M}
\end{array}\right)+L_{k g}\left(\begin{array}{l}
0 \\
0 \\
g
\end{array}\right) \text {, }
$$

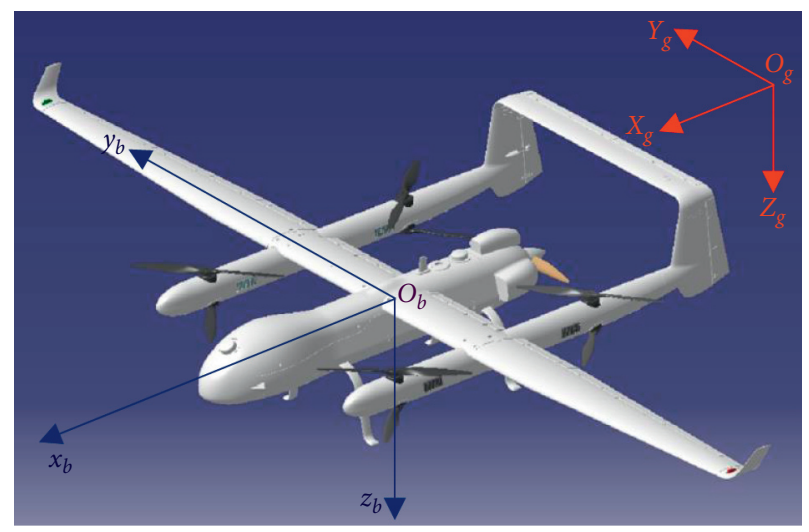

FIgURE 2: SD-60 VTOL UAV.

where $T$ is the engine thrust, $D_{M}$ represents the resultant force of the rotor drag, $L_{M}$ shows the resultant force of the rotor lift that coincides with $Z_{b}$ of the body coordinate, and $D$ and $L$ are the aerodynamic drag and lift forces of the fixed wing, respectively. Moreover, $L_{k a}, L_{k b}, L_{k g}$ denote the conversion matrices from the wind, body, and Earth-surface reference frames to the path coordinate frame, respectively. Then,

$$
\left[\begin{array}{c}
\dot{V} \\
\dot{\chi} \\
\dot{\gamma}
\end{array}\right]=\left[\begin{array}{c}
\frac{\left(T \cos \alpha-m g \sin \gamma-L_{M} \sin \alpha-D-D_{M}\right)}{m}, \\
\frac{\left(T \sin \alpha \sin \mu+L \sin \mu+L_{M} \sin \mu \cos \alpha\right)}{m V \cos \gamma}, \\
\frac{\left(T \cos \mu \sin \alpha-m g \cos \gamma+L_{M} \cos \mu \cos \alpha+L \cos \mu\right)}{m V}
\end{array}\right],
$$

where $\alpha$ is the angle of attack (AoA), $\gamma$ is the track inclination angle (flight path angle), $\chi$ denotes the track azimuth angle, and $\mu$ shows the track bank angle [11].

To determine the UAV's flight trajectory in space, the motion equation of the center of mass needs to be established [12]:

$$
\left\{\begin{array}{l}
\dot{X}_{g}=V \cos \gamma \cos \chi \\
\dot{Y}_{g}=V \cos \gamma \sin \chi \\
\dot{Z}_{g}=-V \sin \gamma
\end{array}\right.
$$

where $X_{g}, Y_{g}$, and $Z_{g}$ are the coordinates in the Earthsurface reference frame with origin $O_{g}$ being located at the launch point on the ground. A complete external force distribution setup is presented in Figure 3, where $O$ is the center of mass of the UAV and $O^{\prime}$ is the projection of $V$ onto the $X_{g} O_{g} Y_{g}$ plane. $O X_{b}$ is the $X$-axis of the body frame, $O X_{b}{ }^{\prime}$ is the projection of $O X_{b}$ onto the horizontal plane, and $\theta$ is the pitch angle satisfying the equation when no roll and no sideslip occur:

$$
\theta=\alpha+\gamma
$$




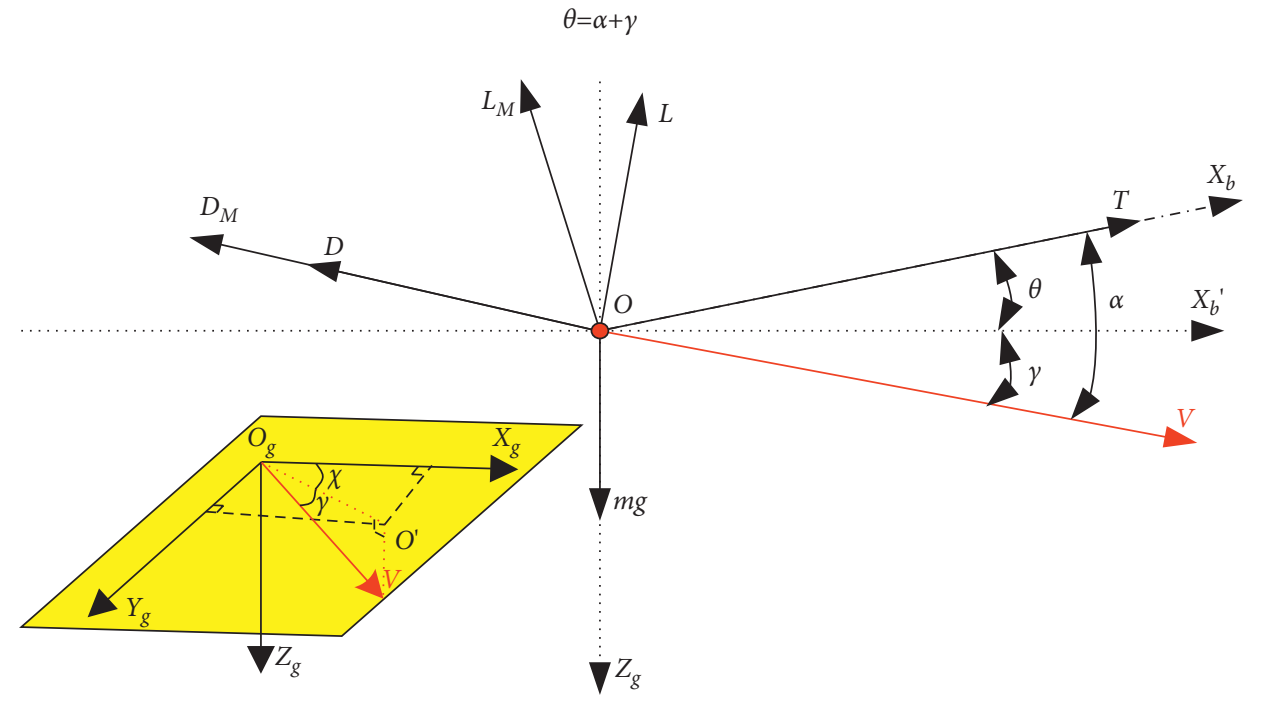

Figure 3: External force distribution.

\section{Aerodynamic Characteristics}

Figure 4 illustrates the aerodynamic analysis employing CFD simulation. The lift coefficient (CL) increases linearly and varies as AoA changes from $-5^{\circ}$ to $12^{\circ}$, with the maximum value close to being 1.76. Figure 4(a) indicates that the critical AoA is $16^{\circ}$, affording a good lift performance.

When Mach number $M_{a}=0.1$, the lift-drag $(L / D)$ characteristics are illustrated in Figure 4(c). Specifically, the maximum lift-drag ratio is close to 14.5 , with the favorable lift coefficient being about 1.2. Combining Figure 4(a) with Figure $4(\mathrm{c})$, the favorable AoA is $4^{\circ}-6^{\circ}$, while when the AoA exceeds $8^{\circ}$, the drag increases sharply.

Figure $4(\mathrm{~d})$ presents the polar curve for Mach number $M_{a}=0.1$ and flight height $H=1000 \mathrm{~m}$. The zero-lift resistance is less than 0.05, and the drag coefficient (CD) corresponding to the maximum lift coefficient is less than 0.15 , highlighting the resistance characteristics of our VTOL UAV.

Accordingly, Figure 4(b) shows that the efficiency of the trim elevator surface is high when the AoA is within $0^{\circ}$ to $6^{\circ}$. Each degree of elevation deviation affords a $0.62^{\circ}$ change in the AoA, ensuring high attitude control accuracy.

\section{Constraints for the States and Controls}

The GPM approximates the state and control variables of the optimal control problem by constructing Lagrange interpolation polynomials. The optimal control problem is transformed into a nonlinear programming (NLP) problem with a series of algebraic constraints by exploiting its derivative to approximate the state's variable derivative concerning time [13]. This section primarily uses GPM to design the VTOL UAV landing trajectory exploiting the GPOPS 5.0 software.

Let the state vector be $x=\left[V, \chi, \gamma, X_{g}, Y_{g}, Z_{g}, E, \theta\right]^{T}$ and the control vector be $u=\left[q, \mu, F_{E}, R_{E}\right]^{T}$, where $E$ is the total energy stored in the VTOL power system, $q$ is the angular pitch rate, $F_{E}$ indicates the throttle value of the cruising engine, and $R_{E}$ is the base throttle value of the rotor motors.

During a regular flight, the states and control of the UAV are within the normal range. However, for a nonstandard flying environment, the scope of some state or control variables may be tightening, for example, when flying without power, the altitude will only decrease or only considers vertical plane motion where the velocity and displacement in the horizontal plane are zero. Furthermore, different trajectories will be obtained under different upper and lower limits of state, control variables, and performance functions, so it is critical to select reasonable upper and lower limits and performance functions according to the task requirements and flight conditions.

4.1. States Limits. In this work, we consider the following states limits:

(1) The experimental UAV approaches from a $50 \mathrm{~m}$ altitude at a cruising speed of $30 \mathrm{~m} / \mathrm{s}$ and lands without horizontal power. Thus, the upper and lower limits of height $\left(Z_{g}\right)$ are $50 \mathrm{~m}$ and $0 \mathrm{~m}$, respectively.

(2) The ideal velocity change gradually decreases as height decreases, and once the UAV touches down, its speed drops to zero. Hence, the upper and lower limits of velocity $(V)$ are $30 \mathrm{~m} / \mathrm{s}$ and $0 \mathrm{~m} / \mathrm{s}$, respectively.

(3) Considering the crosswind case, the UAV flies in the positive or negative direction of the $\mathrm{PE}$ axis after the approach. Thus, the upper and lower limits of the track azimuth angle $(\chi)$ are $90^{\circ}$ and $-90^{\circ}$, respectively.

(4) In the case of no cruising power, the UAV can only change the flight path angle $(\gamma)$ to distribute the component of gravity in the velocity direction, which is the critical factor in controlling the change in speed. So the flight path angle can only be less than 


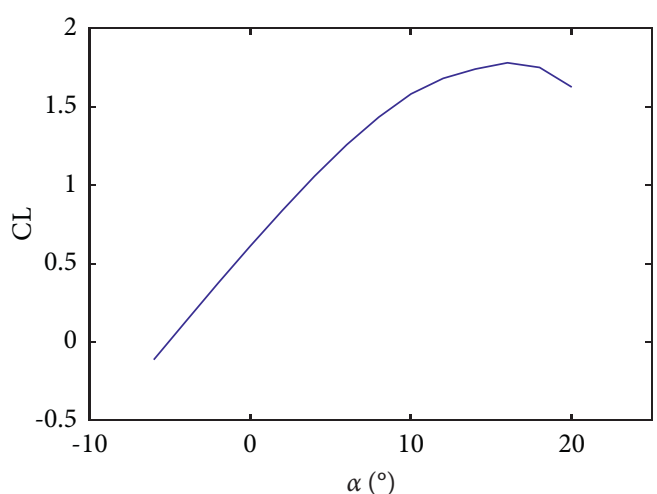

(a)

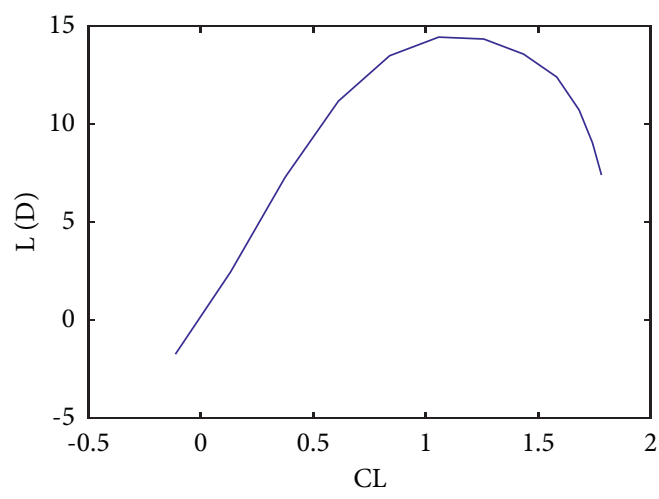

(c)

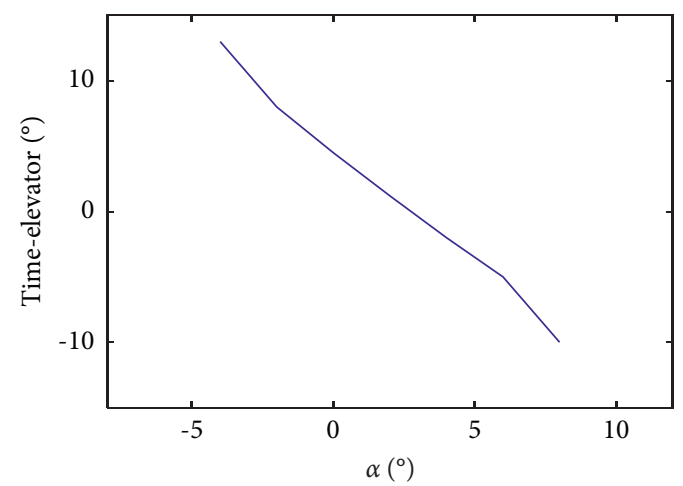

(b)

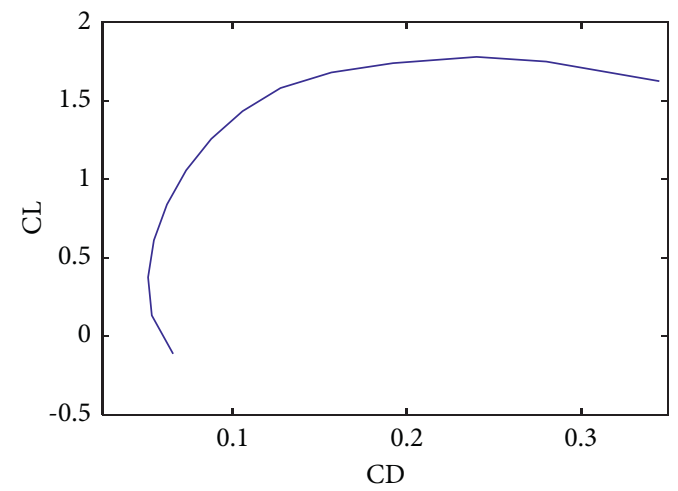

(d)

FIgURE 4: Aerodynamic characteristics.

zero. When the UAV lands vertically, the velocity direction is perpendicular to the horizontal plane, so the flight path angle is at least $-90^{\circ}$.

(5) It is assumed that the UAV flies at a maximum speed of $30 \mathrm{~m} / \mathrm{s}$ for $90 \mathrm{~s}$, and the distance traveled during this time is the maximum flying distance in the direction of the PE or PN axis. Thus, the upper and lower limits of the forward distance $\left(X_{g}\right.$ and $\left.Y_{g}\right)$ are $2700 \mathrm{~m}$ and $0 \mathrm{~m}$, respectively.

(6) When the throttle value $\left(R_{E}\right)$ of the rotor motors is 60 in the multirotor mode, the UAV can hover in a stable state. The total energy $(E)$ is defined as the product of the throttle and the hover time. The total energy stored in the VTOL power system can support the UAV to hover for $90 \mathrm{~s}$. Hence, the upper and lower limits of total energy are 5400 and 0 , respectively.

(7) During level cruising, the UAV's trim pitch angle is $3^{\circ}$. Considering the variation range of the AoA and the flight path angle, using equation (5), we calculate the upper and lower limits of the pitch angle $(\theta)$ to be $12^{\circ}$ and $-10^{\circ}$, respectively.

Depending on the flight phase, that is, start, intermediate, and terminal, Table 1 defines the upper and lower limits of several states.
TABLE 1: Lower and upper state limits per trajectory phase.

\begin{tabular}{lccc}
\hline \multirow{2}{*}{ State } & \multicolumn{3}{c}{ The lower and upper state limits } \\
& Starting $\left(t_{0}\right)$ & Intermediate $(t)$ & Terminal $\left(t_{f}\right)$ \\
\hline$V$ & {$[30,30]$} & {$[0,30]$} & {$[0,0]$} \\
$\chi$ & {$[0,0]$} & {$[-(\pi / 2),(\pi / 2)]$} & {$[-(\pi / 2),(\pi / 2)]$} \\
$\gamma$ & {$[0,0]$} & {$[-(\pi / 2), 0]$} & {$[-(\pi / 2), 0]$} \\
$X_{g}$ & {$[0,0]$} & {$[0,2700]$} & {$[0,2700]$} \\
$Y_{g}$ & {$[0,0]$} & {$[0,2700]$} & {$[0,2700]$} \\
$Z_{g}$ & {$[50,50]$} & {$[0,50]$} & {$[0,0]$} \\
$E$ & {$[5400,5400]$} & {$[0,5400]$} & {$[0,5400]$} \\
$\theta$ & {$[3,3]$} & {$[-10,12]$} & {$[0,0]$} \\
\hline
\end{tabular}

4.2. Controls Limits. Setting lower and upper limits on the controls during the trajectory phases is also essential. Based on a large amount of test flight data and experience, the upper and lower limits of the angular pitch rate $(q)$ can be obtained between $-5 \%$ and $5 \%$. Due to the large roll angle, the track bank angle $(\mu)$ is limited to $\pm 30^{\circ}$ to prevent lift loss and attitude reversal. To simulate no cruise power, the throttle of the cruise engine $\left(F_{E}\right)$ is always zero. In the multirotor mode, attitude control is achieved by the rotational speed differential of each motor over the base throttle $\left(R_{E}\right)$. At least $10 \%$ of the differential control variable must be reserved for attitude control so that the maximum throttle of each motor is $90 \%$. Hence, 


$$
\begin{aligned}
\frac{-5^{\circ}}{s} \leq q & \leq \frac{5^{\circ}}{\mathrm{s}} \\
-30^{\circ} & \leq \mu \leq 30^{\circ}, \\
0 & \leq F_{E} \leq 0, \\
0 & \leq R_{E} \leq 90 \% .
\end{aligned}
$$

4.3. Path Constraints. According to Figure 4, the optimum AoA for the fixed-wing mode is within $-5^{\circ}$ to $15^{\circ}$. However, the AoA can reach $90^{\circ}$ during vertical landing, where the aerodynamic force is almost zero in the multirotor mode. This upper limit is very critical. The AoA of a conventional fixed-wing aircraft must be limited to stall AoA; otherwise, it will crash due to lift loss. But a VTOL UAV can reach an AoA of up to $90^{\circ}$ for a smooth vertical landing because of the extra lift provided by the rotors. The maximum sink rate is limited to $4 \mathrm{~m} / \mathrm{s}$, depending on the glide performance, the aircraft's structural strength, and the maximum overload it can withstand. Hence, the path constraints during this phase are

$$
\begin{aligned}
-5^{\circ} & \leq \alpha \leq 90^{\circ} \\
0 & \leq \dot{Z}_{g} \leq 4 \frac{\mathrm{m}}{\mathrm{s}} .
\end{aligned}
$$

4.4. Cost Function. The cost function contains the endpoint and integrand cost. Setting the endpoint cost function $(\Phi)$ depends on the optimal control problem examined; for example, for the minimum time-to-land problem, the endpoint cost is flight time, while for an emergency landing, the endpoint cost function is the forward flight distance. $\Phi$ is a function of the terminal value of the flight time or the forward flight distance $\left(X_{f}\right)$. Overall, the endpoint cost function is given as

$$
\begin{aligned}
\Phi & =\Phi\left[t_{f}\right], \\
\operatorname{or} \Phi & =\Phi\left[X_{f}\right] .
\end{aligned}
$$

To stabilize the UAV's attitude and smooth the flight path, we set the integral term of the integrated cost function $(J)$ as the sum square of the sink rate and the angular rate of the flight path angle [14].

$$
J=\int_{t_{0}}^{t_{f}}\left(k_{1} \dot{Z}_{g}^{2}+k_{2} \dot{\gamma}^{2}\right) \mathrm{d} t,
$$

where $k 1$ and $k 2$ are the weight coefficients. The greater the value, the greater the penalty, indicating the higher attention given to the corresponding item.

\section{Trajectory Planning}

The typical landing process of a wheeled fixed-wing UAV includes three stages: approach glide, flare landing, and taxi $[15,16]$. These stages are depicted in Figure 5 as trajectory

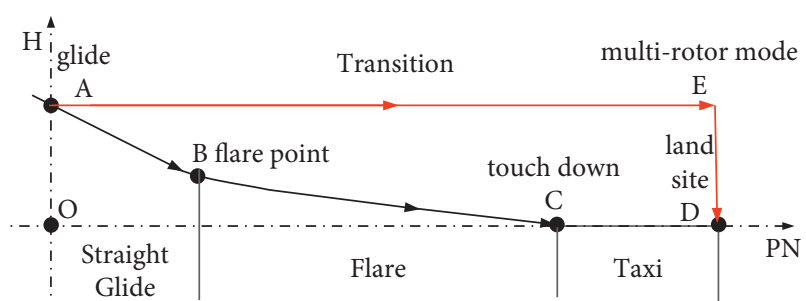

FIgURe 5: Typical landing trajectory (ABCD is for typical fixedwing UAVs and AED for VTOL UAVs).

$\mathrm{ABCD}$, with the AED trajectory being the corresponding one for a VTOL UAV. To mathematically describe the landing trajectory, we utilize the Northeast coordinate system, where the origin point $\mathrm{O}$ is the landing window point, the positive direction of the $\mathrm{PN}$ axis points to the North direction, and the positive direction of the PE axis points to the East direction.

5.1. Emergency Landing. A VTOL UAV has its own engine shutdown forced landing characteristics due to the VTOL power system. The prominent feature of such power systems is not being limited by airport conditions, as a relatively open ground near the route is adequate for an emergency landing. Another feature involves the potential energy from the engine shutdown point to the nearest crash-landing point being generally rich. Therefore, excess energy dissipation should be considered. Finally, after entering the landing window, the UAV can either land in a multirotor mode or be assisted by the rotary-wing lift and perform glide landing at a lower forward speed and sink rate.

In terms of a VTOL UAV, the proposed landing phases comprising the entire process from the engine shutdown position to the landing site are guidance, spiral, and landing. The entire trajectory is illustrated in Figure 6.

5.1.1. Guidance Phase (A-B). Given that the initial UAV position and attitude are arbitrary, guiding the UAV towards the landing window is necessary. Hence, we employ the Dubins shortest cross-range method for the lateral trajectory, which consists of the arc $A A^{\prime}$, arc $B^{\prime} B$, and line $A^{\prime} B^{\prime}$ (see Figure 7), and for the vertical, we define the height profile exploiting the sliding performance constraints [17].

While flying in circles, the UAV's descending height is proportional to the radius of the arc and the flight path angle:

$$
\triangle h=2 \pi R \tan \gamma .
$$

In the vertical plane, the UAV is in an equilibrium sliding state, and thus equation (10) can be obtained:

$$
\frac{1}{\tan |\gamma|}=\frac{L}{D} \text {. }
$$

When the lift-drag ratio $L / D$ is maximum, the flight path angle is the shallowest, and the descent height for a given flight distance is minimum.

Generally, the emergency landing site of a VTOL UAV is near the engine shutdown position. Hence, to reduce the 


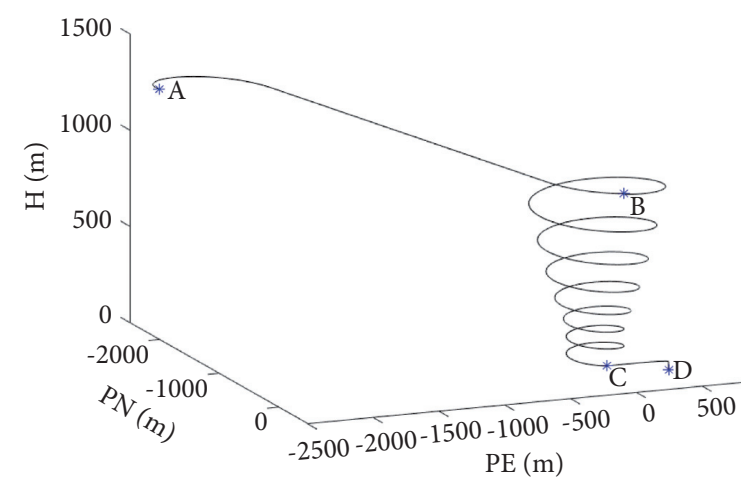

Figure 6: Complete landing trajectory for a VTOL UAV.

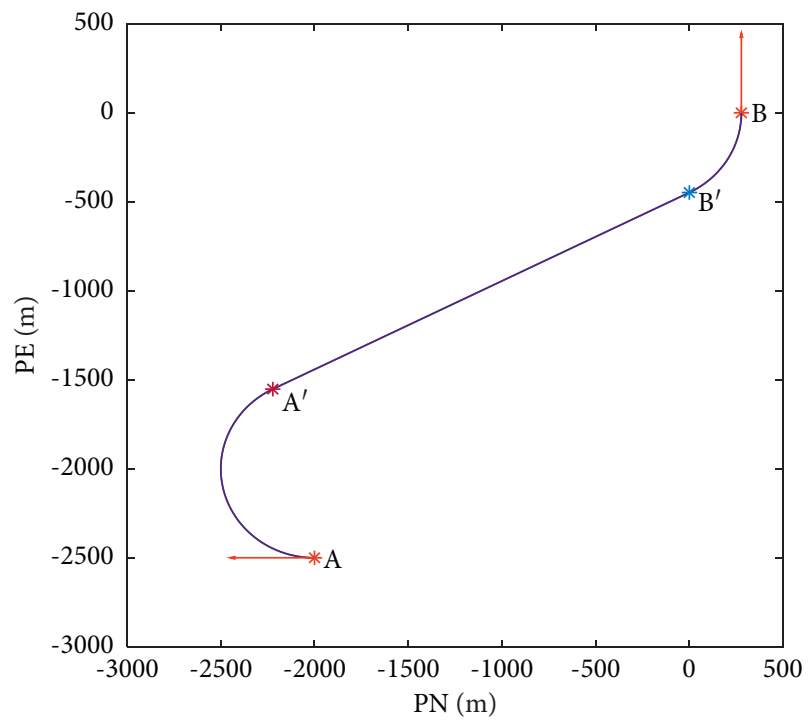

FiguRE 7: Dubins shortest cross-range trajectory.

UAV's height as quickly as possible, the radius of $\operatorname{arc} A A^{\prime}$ and $B^{\prime} B$ for the heading angle adjustment should be large, and the maximum flight path angle should be appropriately adjusted during the straight glide phase $A^{\prime} B^{\prime}$.

5.1.2. Spiral Trajectory Phase (B-C). In this trajectory phase, the UAV spirals down, not only adjusting its heading angle but dissipating the potential excess energy. A large roll angle results in excessive lifting force loss during the spiral motion, and a significant acceleration overload may damage the body structure. In this phase, the UAV glides are in balance with the hovering radius given by

$$
R=\frac{V^{2} \cos \gamma}{g \tan \phi} .
$$

According to equations (9) and (11), to reduce height as quickly as possible in the initial stage, the UAV requires a large spiral radius, and thus a smaller roll angle is recommended. However, as the UAV's height decreases, the radius decreases until the minimum allowable hover radius is reached. If the flight altitude is still above the landing window, the UAV will continue to spiral down from this minimum radius. The higher the altitude is, the fewer obstacles the UAV encounters, and thus the spiral radius can be more extensive. However, if obstacles are present, the radius must be strictly limited. To reduce the height as soon as possible, a conical helix (blue curve in Figure 8) with a larger radius is designed at the top of the spiral section, while a cylindrical spiral trajectory (red curve in Figure 8) with a fixed smaller radius (the minimum radius) is designed at the bottom. In the cylindrical spiral trajectory, for the UAV flying the same number of circles, the smaller the minimum radius, the least the height reduction. Increasing or decreasing the number of circles makes it possible to fine-tune the height at a high resolution and adjust the heading angle in the range of $0-360^{\circ}$, which is conducive to the UAV's states to meet the approach requirements.

The recommended heading angle for waypoint $\mathrm{C}$ is usually against the wind direction, and the air corridor should be empty in this direction.

The initial heading angle, position, and altitude of the $\mathrm{UAV}$ at the engine shutdown point $\mathrm{A}$ will affect the trajectory of the guidance and the spiral section (see Figures 9-11). Indeed, the simulation results verify that the proposed strategy can safely guide the UAV to the landing window and meet the heading and altitude requirements for a safe landing.

5.1.3. Landing Phase (C-D). The VTOL power system affords the UAV to perform various landing trajectories once it enters the landing window. The trajectory is related to the energy consumption rate, duration of action, and the multirotor system's force magnitude. Once the UAV concludes the B-C spiral trajectory and passes endpoint $\mathrm{C}$, the UAV's heading angle meets the landing requirements, and the path planning focuses on the longitudinal trajectory.

\section{Case 1. Conventional landing}

When the height and wind direction meet the landing requirements and the energy storage of the VTOL system is sufficient, the UAV can land conventionally. For a control variable $\mu=0$, the landing trajectory in the vertical plane is shown in Figure 12.

The UAV raises its head to increase the pitch angle and uses drag to decelerate. At this phase, the multimotor mode is activated to provide lift, allowing the UAV to slow down while still flying flat (see Figure 13). The forward velocity continues to decrease until it becomes zero, and once the UAV completes the transition from the fixed-wing mode to the multirotor mode, it then lands vertically.

The maximum sink rate is $2.0 \mathrm{~m} / \mathrm{s}$. The rotors operate throughout the landing process and consume only $60 \%$ of the VTOL energy, guaranteeing the UAV to land safely under normal conditions. By optimizing the trajectory, the UAV can reduce battery consumption during landing, increase the power margin, and improve safety landing.

\section{Case 2. Forced landing}

Once the UAV enters the landing window, it follows the forced landing trajectory if the multirotor system fails. The 


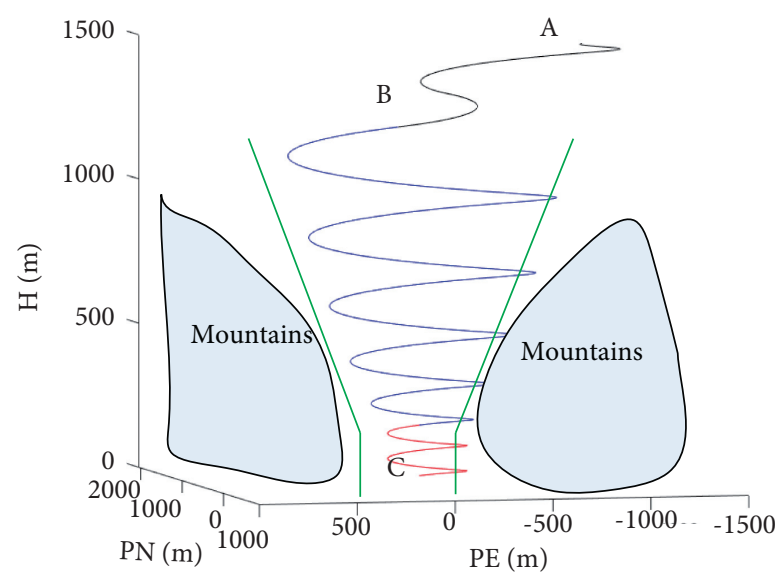

Figure 8: Spiral down trajectory.

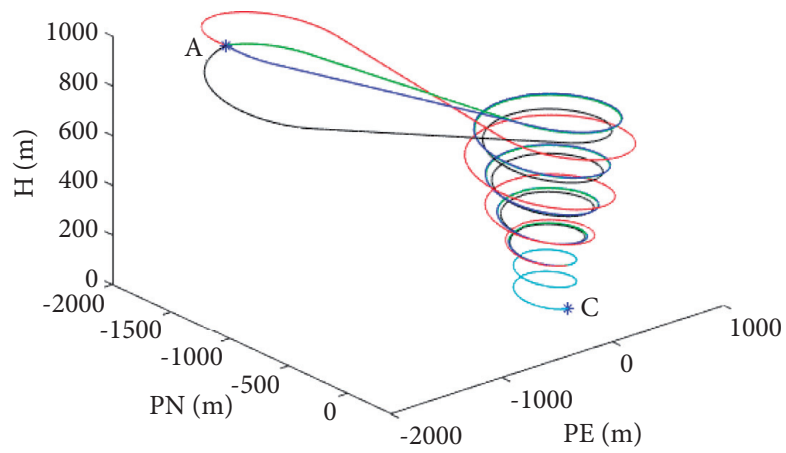

FIgURE 9: Trajectories vary with heading angle.

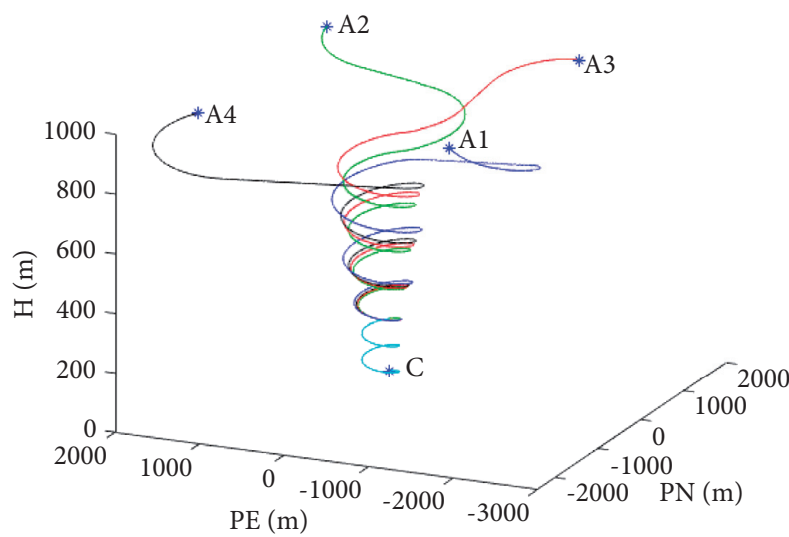

Figure 10: Trajectories vary with heading angle and altitude.

VTOL UAV has no wheels and thus cannot glide once the ground is touched. Therefore, it has to land at a low forward velocity and sink rate. For control variables $\mu=0$ and $R_{E}=0$, Figure 14 shows the forced landing trajectory in the vertical plane.

The UAV increases the gravity component in the velocity direction by increasing the flight path angle (Figure 15), affording sufficient flight velocity. Most of the flight time involves an AoA close to the favorable AoA, while the flight path angle is within $4^{\circ}$. The height descent is relatively gentle, with a maximum sink rate of $1.8 \mathrm{~m} / \mathrm{s}$. Finally, the UAV touches the ground at a forward speed of $22 \mathrm{~m} / \mathrm{s}$ and a sink rate of $0.6 \mathrm{~m} / \mathrm{s}$. Despite the controlled landing trajectory, there is still a high probability of damaging the airframe [18].

Case 3. Distance optimization landing

Given that the VTOL power system has limited energy storage capability, it is necessary to reasonably plan the landing trajectory according to the forward flight distance. The upper and lower boundaries of the landing sites 


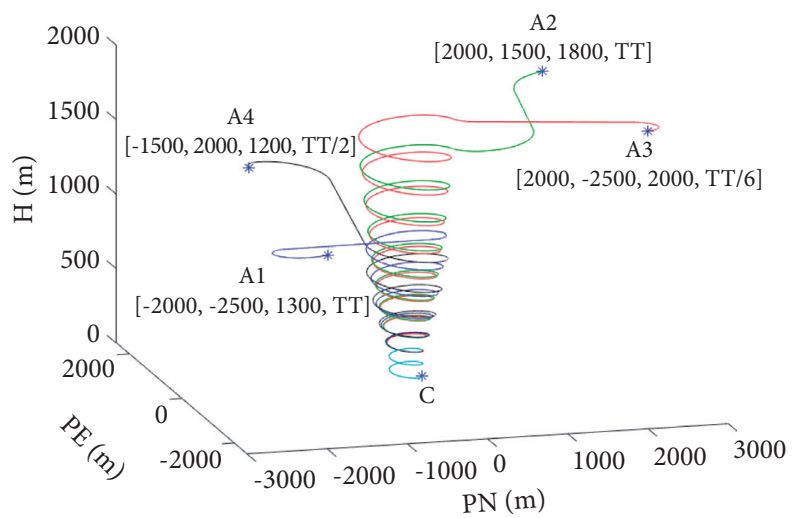

FIGURE 11: Trajectories vary with heading angle, position, and altitude.

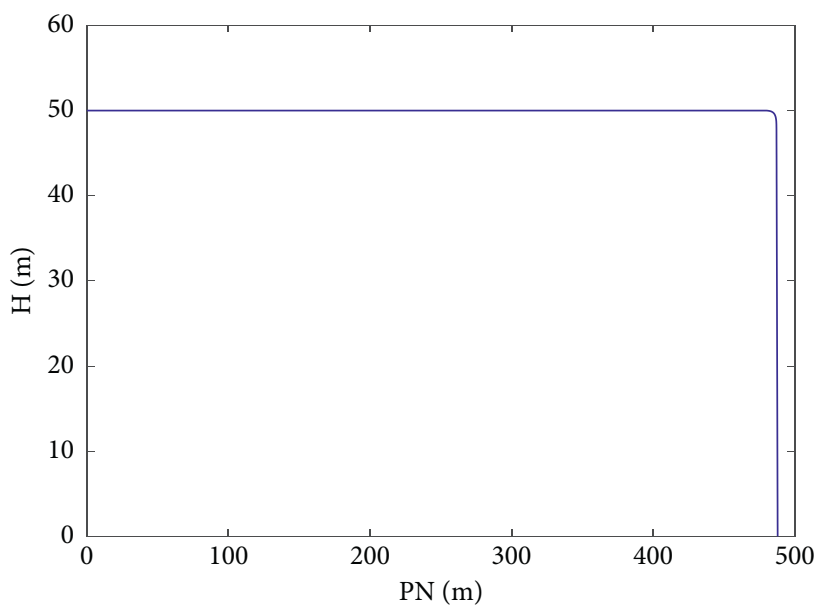

Figure 12: Longitudinal trajectory.

correspond to the furthest and nearest forward flight distance trajectories, respectively. Our experiment's nearest forward distance is only $250 \mathrm{~m}$, and the trajectory is relatively smooth without fluctuations (Figure 16).

Through comprehensive analysis of Figure 17, it is concluded that as velocity decreases, the UAV increases the AoA and the flight path angle and preserves a constant maximum sink rate of $4 \mathrm{~m} / \mathrm{s}$. As it approaches the ground, the UAV reduces its sink rate by reducing the flight path angle and finally touches the ground smoothly under the control of the multirotor mode.

To minimize the forward distance, the UAV descends at the given upper limit of the sink rate, and the rotor force acts primarily as a drag in the opposite direction of the velocity. The VTOL energy consumption is only $18 \%$ affording a large energy margin.

Figure 18 illustrates the farthest forward distance trajectory, highlighting that the UAV maintains level flight for a while before descending. The flight path angle is minimal throughout this stage, close to $3^{\circ}$, with a maximum sinking rate of $1.1 \mathrm{~m} / \mathrm{s}$ (Figure 19). The rotor force vector provides forward flight power, and the velocity is controlled by changing the pitch angle. The UAV pulls up at the end of the shallow glide, rapidly reducing forward velocity and sink rate. The overall landing process is very smooth.
The farthest forward distance is $2249 \mathrm{~m}$, and the energy consumption is $100 \%$. To some extent, the amount of energy stored determines the forward distance of the trajectory [19]. When the height of point $\mathrm{C}$ (center of the landing window) is $50 \mathrm{~m}$, the forward distance is between $250 \mathrm{~m}$ and $2249 \mathrm{~m}$ (Figure 20). The remaining height of the spiral section that cannot reduce through one complete circle is added on the landing section as the height of point C's upper and lower boundaries, which are between $20 \mathrm{~m}$ and $80 \mathrm{~m}$. Therefore, adequate distance from the landing window to the landing site should be considered [20].

\section{Case 4. Wind resistant landing}

If the wind direction changes during landing, the UAV should have a particular lateral control ability to ensure that it lands against the wind. The magnitude of the maximum bank angle is limited to $30^{\circ}$ and the control variable is $\mu=[-30,30]$. Assuming that the incoming airflow direction is distributed between $-90^{\circ}$ and $+90^{\circ}$, the angular value of the incoming airflow direction is obtained every $10^{\circ}$ to estimate the optimal distance trajectories.

Figures 21 and 22 illustrate the landing site distribution under the action of lateral-directional control after the upwind direction changes. The green area indicates the high probability landing area. For the boundary trajectories, such 

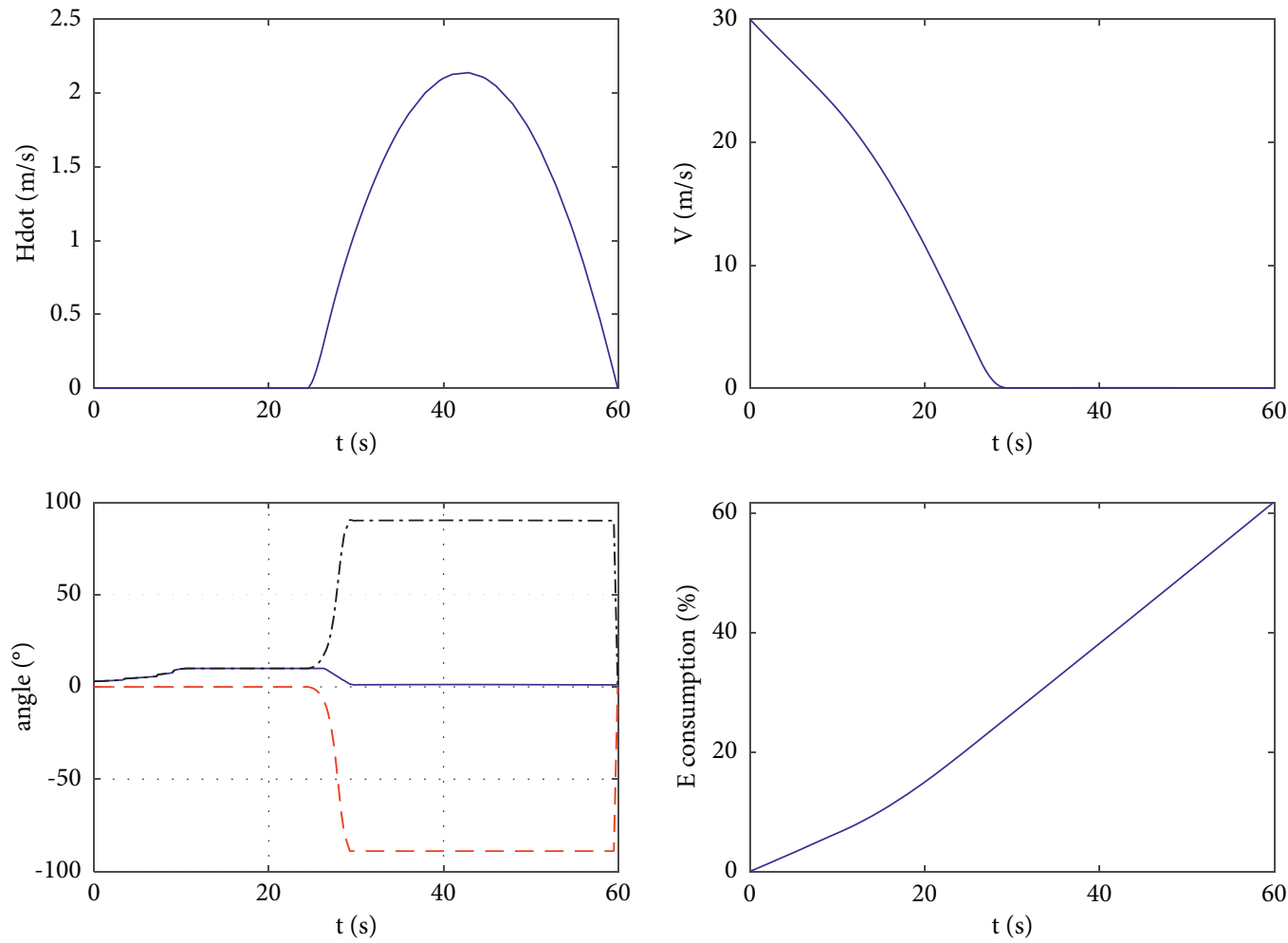

$-\theta$
$--\gamma$
.$- \alpha$

FIgURE 13: States of the conventional landing.

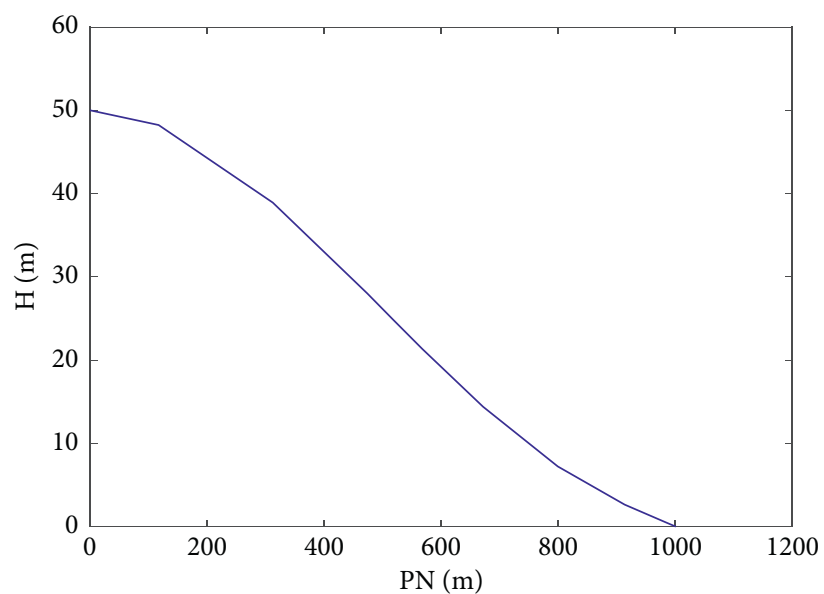

FIGURE 14: Forced landing trajectory. 

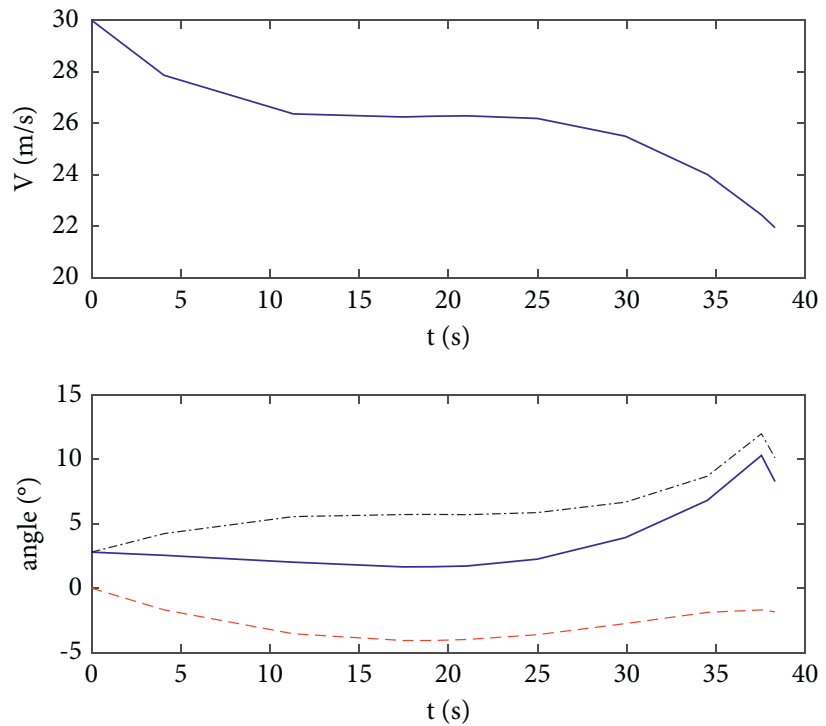

$$
\begin{aligned}
& -\theta \\
& --\gamma \\
& -. \quad \alpha
\end{aligned}
$$

FIGURE 15: States of a forced landing.

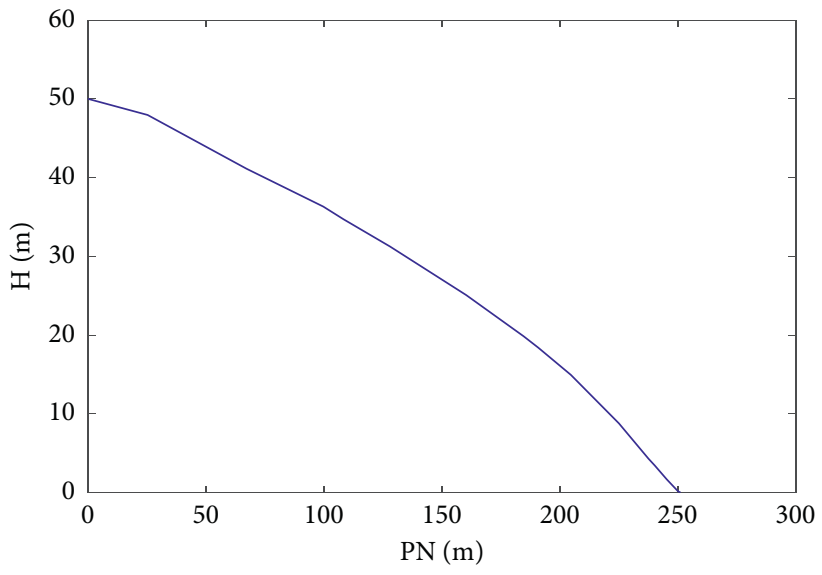

Figure 16: The nearest longitudinal trajectory. 

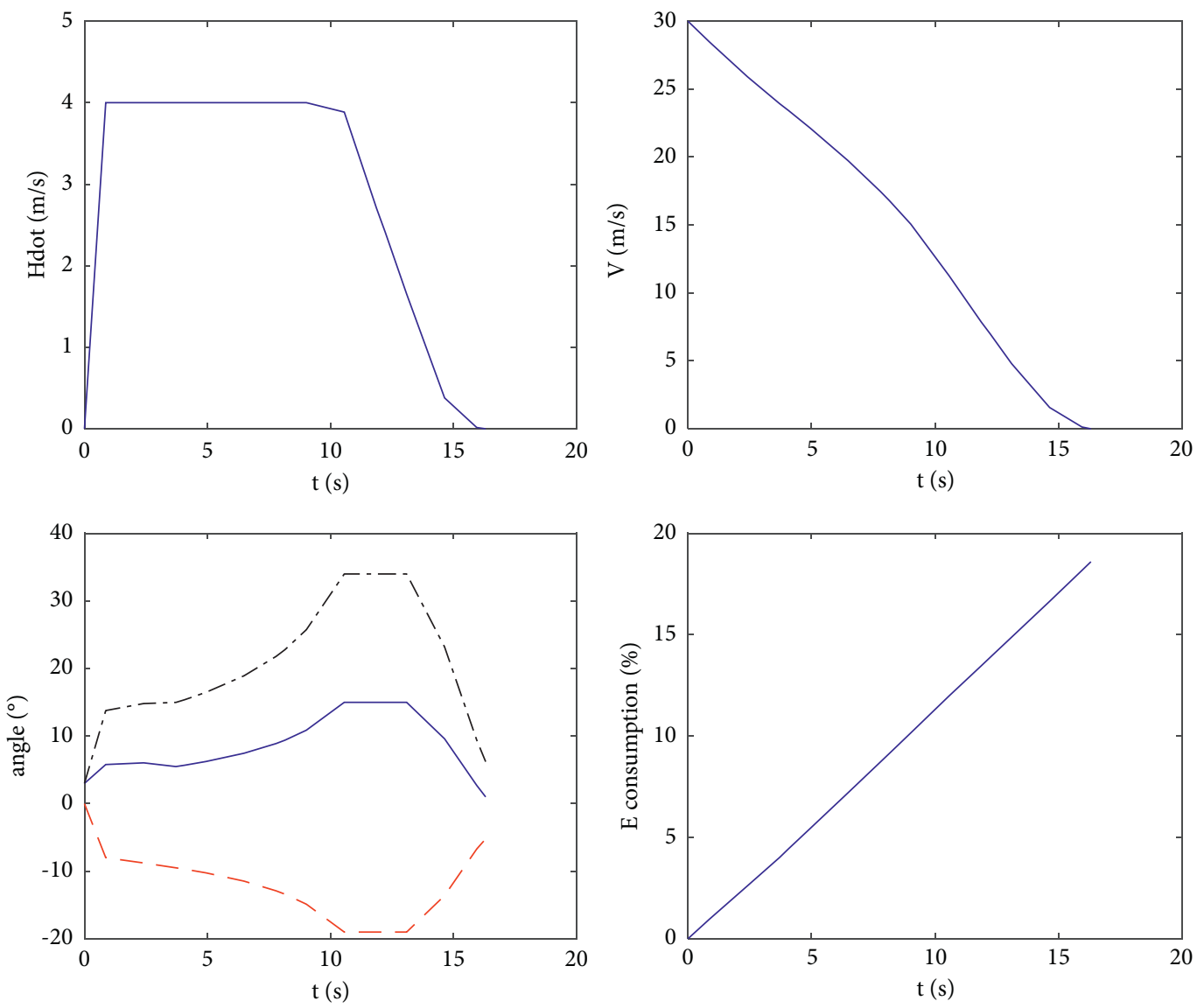

$-\theta$
$--\gamma$
$\ldots \alpha$

FIgURE 17: Nearest trajectory states.

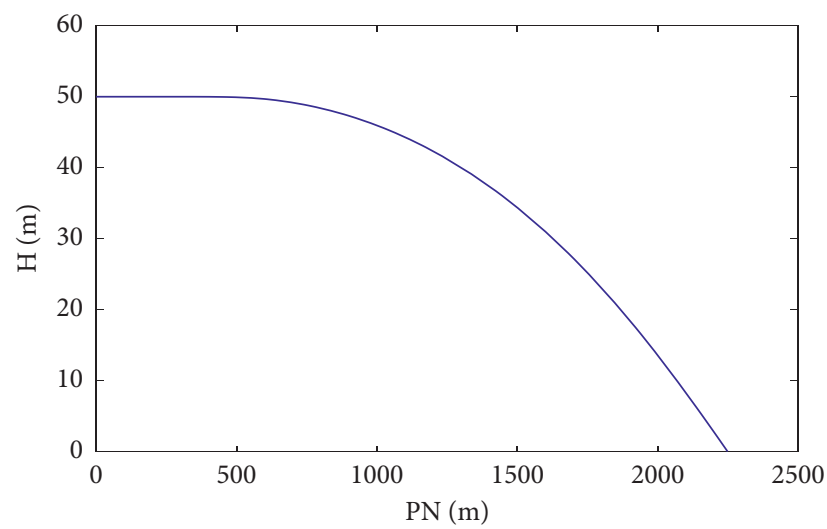

FIgURE 18: The farthest longitudinal trajectory. 

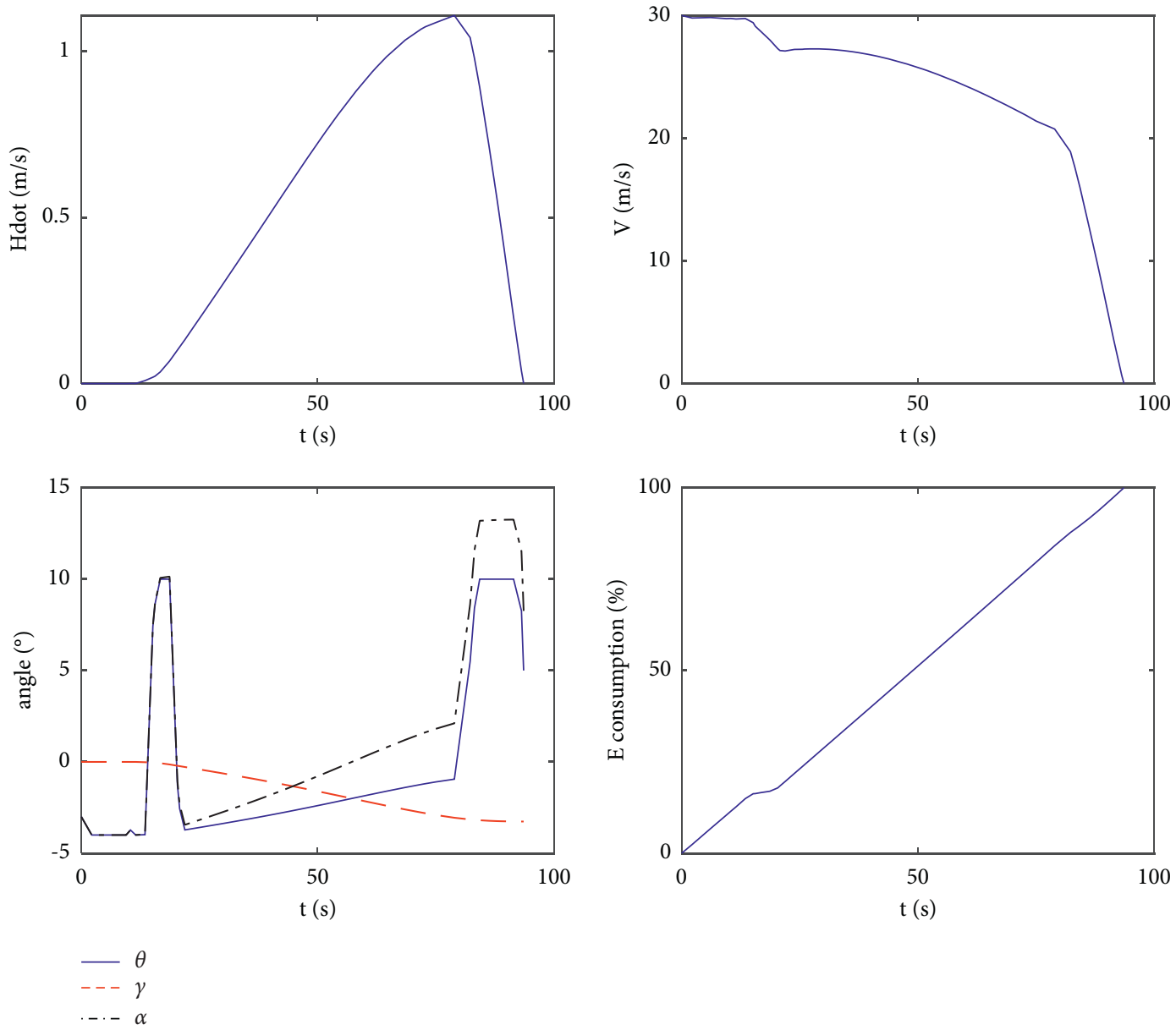

Figure 19: Farthest trajectory states.

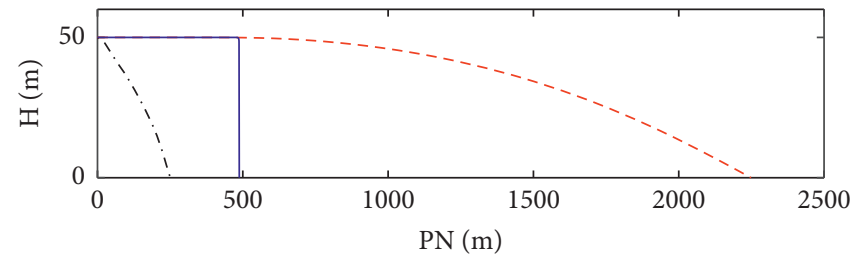

... the nearest trajectory

- - - the farthest trajectory

— the normal trajectory

FIGURE 20: Three typical trajectories. 


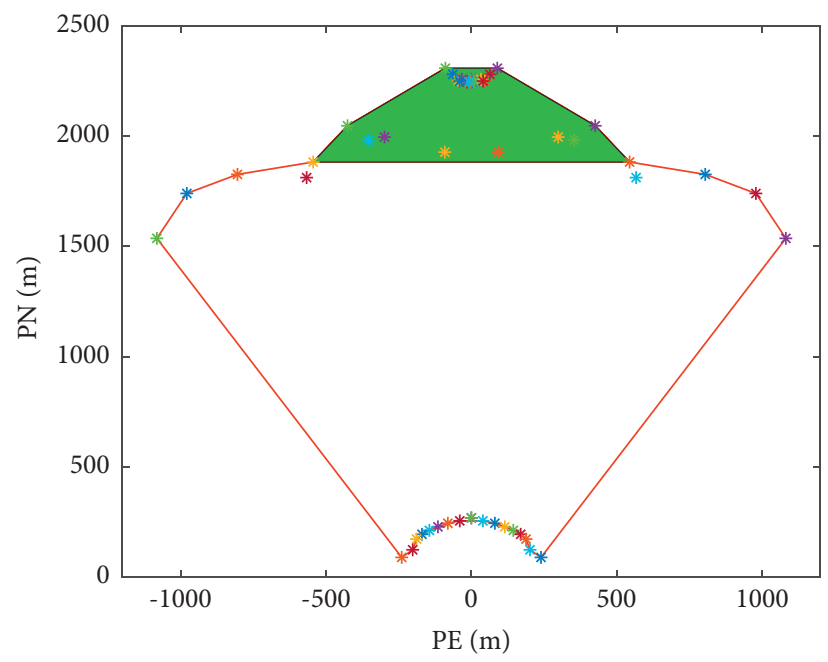

Figure 21: Landing sites distribution.

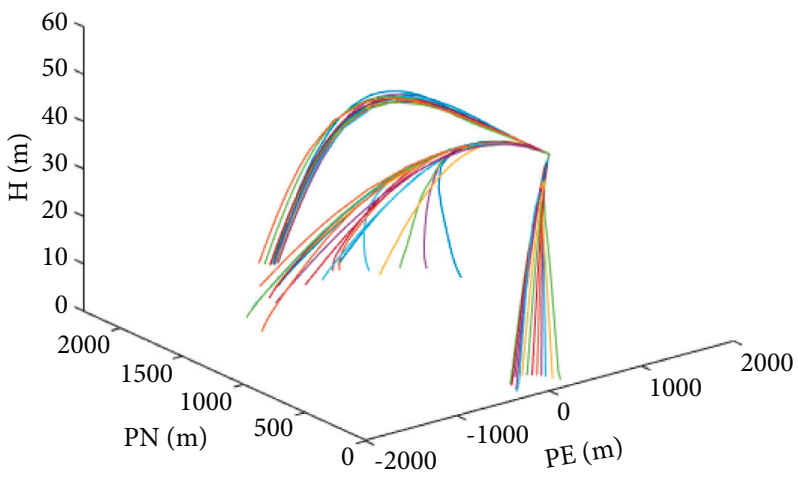

FIGURE 22: Wind-resistant landing trajectories.

as the farthest and the nearest trajectories, the distribution of the landing sites is more concentrated and less affected by the upwind direction [21].

\section{Conclusion}

This paper focuses on the emergency landing trajectory of a VTOL fixed-wing UAV. Hence, it proposes a spiral descent trajectory with variable radius, affording energy management and course adjustment in the spiral section, under the condition of limited energy storage of the VTOL power system. Our trials include several landing trajectories, conventional, unpowered, distance optimal, and wind-resistant landing, which are planned based on the Gauss pseudospectral method applied within the landing section. The simulated trajectories demonstrate the capabilities of our method, which meets the dynamics, terminal, and sliding performance constraints and affords an appealing solution for VTOL fixed-wing aircraft.

6.1. Limitations. VTOL fixed-wing UAV is a very complex combination because of the aerodynamic coupling between the rotor system and the fixed-wing system, and it is difficult to describe it with accurate mathematical model. The landing trajectory proposed in this paper is largely dependent on its aerodynamic characteristics, so the aerodynamic characteristics of the assembly limit the usability of the trajectory to some extent. On the other hand, after the UAV enters the landing window, it follows the forced landing trajectory if the multirotor system fails, so there is always a chance of forced landing as well.

6.2. Future Scope. At present, VTOL fixed-wing UAVs include tail-sitter UAV, tilt-rotor UAV, tilt-wing UAV, and fixed-rotor UAV. The emergency landing trajectory proposed in this paper is mainly suitable for the safe landing of fixed-rotor VTOL fixed-wing UAVs in different landing environments. In the future, suitable emergency landing trajectories will be further studied based on the aerodynamic layout, flight mechanics characteristics, and power configuration of the first three types of UAVs.

\section{Data Availability}

The data used to support the findings of this study are included within the article, but sample data are available on request. 


\section{Conflicts of Interest}

The authors declare that they have no conflicts of interest.

\section{References}

[1] G. Wu, B. Zhou, and L. Yang, "Review and prospect of domestic and foreign civil UAV industry development," Economic Research Guide, vol. 12, pp. 160-162, 2016.

[2] K. Kodera and M. Miwa, "Development of tailsitter VTOL using multi rotor system," The Proceedings of JSME Annual Conference on Robotics and Mechatronics, vol. 1P2-E05, 2017.

[3] K. Dalamagkidis, "Aviation history and unmanned flight," Handbook of Unmanned Aerial Vehicles, Springer Netherlands, Dordrecht, Netherlands, pp. 57-81, 2015.

[4] G. Chen, Y. H. Jia, D. 1. Ma, X. L. Xia, and X. Zhang, "Optimal design approach of series hybrid-electric system for unmanned convertiplane," Journal of Beijing University of Aeronautics and Astronautics, vol. 12, pp. 160-162, 2016.

[5] H. Zhang, B. Song, and H. Wang, "Modeling and optimal design of power system for electric fixed-wing quadrotor hybrid unmanned aerial vehicle," Journal of Aerospace Power, vol. 34, no. 6, pp. 1311-1321, 2019.

[6] J. Sun, X. Pan, and Y. Zhao, "Initial descent phase trajectory planning for near-space glide vehicle," Tactical Missile Technology, vol. 5, pp. 114-120, 2020.

[7] S. Wang, D. Ma, Y. Muqing, and Z. Liang, "Three-dimensional optimal path planning for high-altitude solar-powered UAV," Journal of Beijing University of Aeronautics and Astronautics, vol. 45, no. 5, pp. 936-943, 2019.

[8] Y. Zhang, Research of Unpowered Emergency Landing Control Technologies for UAV, Nanjing University of Aeronautics and Astronautics, Nanjing, China, 2017.

[9] Z. A. Wang, Z. Gong, Y. L. Chen, S. Zhiwei, and X. Jinfa, "Online path planning and guidance for hybrid VTOL UAVs forced landing," Acta Aeronautica et Astronautica Sinica, vol. 40, no. 10, Article ID 323105, 2019.

[10] Z. Fang, Flight Dynamics of Aircraft, Beijing University of Aeronautics and Astronautics Press, Beijing, China, 2005.

[11] S. F. Guo, G. Z. Shen, and C. F. Wu, Advanced Flight Control System, National Defense Industry Press, Arlington, Virginia, 2003.

[12] Q. Quan, Introduction to Multicopter Design and Control, Publishing House of Electronics Industry, Beijing, China, 2018.

[13] A. V. Rao, D. Benson, C. L. Darby et al., "User's manual for gpops version 5.0: a matlab software for solving multiplephase optimal control problems using hp-adaptive pseudospectral methods," 2011.

[14] C. Liu, Constrained Flight Optimal Path Planning for a Vertical Take-Off and Landing Fixed Wing Aircraft, Shenyang Aerospace University, Shenyang, China, 2018.

[15] Z. G. Tian, H. Y. Liu, X. Y. Huang, and H. J. Guo, "Research on unpowered emergency landing control strategy of UAV," Computer Simulation, vol. 36, no. 2, pp. 35-39, 2019.

[16] G. M. Tsikalas, "Space shuttle autoland design," in Proceedings of the American Institute of Aeronautics and Astronautics Guidance and Control Conference, San Diego, CA, USA, August 1982.

[17] W. Liu and S. Kepu, "Research on technology of unpowered glide for vehicles," Computer Simulation, vol. 30, no. 8, pp. 65-69, 2013.
[18] F. Liu and X. Ma, "Simulation research of deep stall landing for electric-powered UAV," Flight Dynamics, vol. 29, no. 5, pp. 77-80, 2011.

[19] S. Hu and Y. Chen, "Application analysis of pseudo-spectral method in trajectory optimization of aircraft," Rocket Propulsion, vol. 40, no. 5, pp. 61-68, 2014.

[20] Y. Enmi, G. Tang, and C. Lei, "Fast reentry trajectory optimization of hypersonic vehicle based on gauss pseudospectral method," Journal of Aerospace, vol. 29, no. 6, pp. 1766-1772, 2008.

[21] T. Antonios and W. Brian, Cooperative Path Planning of Unmanned Aerial Vehicles, National Defense Industry Press, Arlington, Virginia, 2013. 